\title{
PÉREZ SÁINZ, JUAN PABLO. (ED.). VIDAS SITIADAS. JÓVENES, EXCLUSIÓN LABORAL Y VIOLENCIA URBANA EN CENTROAMÉRICA. SAN JOSÉ: FLACSO, 2018
}

Magdalena Madrigal Herrera

Recibido: 11/09/19 - Aceptado: 19/09/19

Vidas sitiadas es un libro publicado por FLACSO sede académica Costa Rica, editado por el investigador Juan Pablo Pérez Sainz, en este libro participan como autores y autoras de los diferentes capítulos, Francisco Hernández Ulloa, Wendy Alas Velado, Margarita Montoya Hernández, Minor Mora Salas y Florencio Ceballos Schaulsonhn.

El documento corresponde a los resultados del proyecto de investigación denominado "Entre la violencia y el empleo. Los dilemas de jóvenes de comunidades urbanas marginales en Centroamérica", que se desarrolló de manera conjunta entre FLACSO Costa Rica y FLACSO El Salvador.

El texto consta de cinco capítulos, que comparten tres ejes orientadores para el análisis: violencia, empleo y juventud. El primero realiza una contextualización de los datos arrojados por la investigación en las comunidades de Los Guido en Costa Rica y Soyapango en El Salvador. El texto avanza con un segundo y tercer capítulo donde se analizan las problemáticas transversales del estudio, imbricadas al contexto y particularidades de cada comunidad desde el enfoque de trayectorias de vida. Finaliza con un cuarto y quinto capítulo, donde se realiza un análisis sobre los riesgos de exclusión sociolaboral y los desafíos de inclusión social para población joven en contextos de violencia.

En el primer capítulo se reflexiona sobre las relaciones laborales que establecen las y los jóvenes que conforman la población objetivo en ambos países, sus expectativas de futuro y esboza las causas de estigmatización territorial que se analizan en los apartados siguientes.

En el caso del capítulo II, el análisis de las trayectorias de vida de la población joven que habita en el distrito de Los Guido se centra en identificar factores que inciden en la superación o consolidación de procesos de exclusión social, para lo cual se analiza el impacto de diferentes mediaciones como: familia, educación, trabajo, instituciones y pares. Resultan interesante las conclusiones del estudio, donde se menciona 
que las mediaciones familiares y de educación contribuyen a reproducir la exclusión social, mientras que los relacionados con pares o vinculación al empleo se asocian con posibilidades de salidas de la exclusión. En este sentido, se argumenta que en el ámbito familiar se presenta la reproducción de roles tradicionales de género que excluyen a las y los jóvenes de educación y empleo, además, la desestructuración de las familias se asocia con mayor nivel de privaciones económicas, que a su vez contribuye a que las trayectorias educativas de esta población se vean interrumpidas por estas carencias, llevando a que los jóvenes ingresen de manera prematura al mercado laboral para contribuir a la manutención del hogar. Por otro lado, en el caso del empleo y los pares, estos les permiten a las y los jóvenes situarse a cierta distancia espacial y simbólica de su cotidianidad, lo que contribuye a visualizar salidas a los procesos de exclusión social.

El tercer capítulo profundiza en el análisis de las trayectorias de vida de jóvenes de las colonias de Soyapango en El Salvador, mostrando dos hallazgos relevantes, el primero, a pesar de que estos jóvenes comparten un contexto de violencia, marginalidad y abandono estatal, muestran trayectorias laborales distintas, que se mueven entre trabajos categorizados como profesionalizantes, domésticos y vulnerables; pero que presentan un rasgo común, se trata de trayectorias laborales caracterizadas por la precariedad. Otro elemento a resaltar es la adaptación que genera esta población a sus entornos de violencia, lo cual si bien les lleva a vivir en un estado de alerta continuo, no incide decisivamente en una ruptura con el arraigo que presentan a su comunidad. Esto en tanto generan estrategias que les permiten coexistir con estas dinámicas, tales como la lectura y compresión de las dinámicas simbólicas mediante la identificación de fronteras intangibles que determinan la movilidad dentro de cada territorio.

Los capítulos cuatro y cinco se nutren del estudio realizado en Los Guido y en Soyapango, para realizar un análisis de los riesgos de exclusión sociolaboral y los desafíos de inclusión social para población joven en un contexto de violencia social. Con respecto a los riesgos de exclusión, el documento muestra que estos están determinados por variables como: país de residencia, sexo, edad, jefatura de hogar, nivel educativo, desempleo y acceso a seguridad social; cabe mencionar que, dependiendo de cómo se relacionen y vinculen estas variables, las y los jóvenes se sitúan en zonas de riesgo de exclusión, alto, medio o en vulnerabilidad.

En el último capítulo, se analizan los desafíos de la inclusión social, enfatizando que si bien desde las instituciones públicas o las empresas privadas existen iniciativas orientadas a propiciar inclusión social de la población joven en situaciones de vulnerabilidad, estas presentan limitaciones en su alcance, en aspectos como: la oferta de iniciativas de empleabilidad no cubren la demanda de mano de obra juvenil, existe poca vinculación entre la formación y las demandas empresariales, se presentan barreras de ingreso al mercado laboral que estas iniciativas no contemplan, como las estigmatización de esta población por su lugar residencia. Por último, se analizan los obstáculos que enfrenta para ingresar al mercado laboral, como restricciones de 
movilidad espacial asociadas con la localización de sus comunidades de residencia en la periferia de las ciudades, y a lo interno de estas, aspectos de seguridad que limitan la libre movilidad.

En este libro el lector encuentra un trabajo de investigación lúcido y robusto que aporta elementos tanto históricos como actuales para comprender la violencia en el contexto centroamericano; histórico, en la medida que establece líneas para explicar porque estas comunidades presentan segregación socio territorial y altos índices de delincuencia y criminalidad. Y actual, dado que a partir de las subjetividades de las y los jóvenes entrevistados se caracteriza y analiza los procesos de exclusión sociolaboral que enfrenta esta población en su tránsito por el empleo.

El libro busca mostrar lo que aquí denominamos lo distinto en lo común, ya que parte del argumento que la población joven de Los Guido y Soyapango se encuentra en contextos de alta violencia social, comparten entornos donde la seguridad es un asunto individual no garantizado por el estado, lo que sustenta el surgimiento de procesos de estigmatización en torno a su aspecto físico, forma de expresarse y su lugar de residencia, asociados en el imaginario social con riesgo, delincuencia y criminalidad.

En este contexto común, el libro enfatiza que hay elementos diferentes en sus trayectorias de vida como las estrategias de adaptación a los entornos de violencia, intensidad de las carencias económicas, la reproducción tradicional de roles de género en las familias y el circulo de pares; estos factores pueden incidir de manera diferenciada para reforzar procesos de exclusión social o para construir posibles salidas a estos.

El libro aporta un mirada crítica con respecto a la construcción de imaginarios sociales en torno a una población específica y los obstáculos que pueden generar para el ingreso o permanencia en el mercado laboral; se concluye que la asociación directa de juventud y marginalidad social con criminalidad, se debe a la construcción de una imagen social simplista que produce supuestos sociales sobre cómo se ven las personas que habitan estas comunidades, como hablan, las actividades económicas a las que se dedican y el estilo de vida que tienen, además dictan que tipo de relaciones conviene establecer con estas.

Finalmente, se puede concluir que este libro contribuye al análisis crítico y compresión del fenómeno complejo de la exclusión social. Con una estrategia metodológica que a partir del trabajo empírico da rostro a esta problemática, lleva al lector a comprender porque se puede decir que las vidas de estos jóvenes están sitiadas y que la estructura social asume el rol de los sitiadores. Además, invita a problematizar la noción de inclusión, enfatizando que no se trata de un estar dentro o fuera, que la inclusión no está determinada exclusivamente por la vinculación al mercado laboral, se trata de un proceso que no es lineal, y donde se ven implicadas otras esferas de lo social, como lo económico, cultural y el género, que conforman obstáculos o barrera de acceso y/o permanencia en el trabajo. 
Magdalena Madrigal Herrera. Costarricense, obtuvo su maestría en Gestión Pública por la Universidad Autónoma de Barcelona, España y la licenciatura en Sociología por la Universidad de Costa Rica. Se desempeña como profesora en la Escuela de Sociología de la Universidad de Costa Rica y como investigadora para la Facultad Latinoamérica de Ciencias Sociales (FLACSO), sede académica Costa Rica.

Correo electrónico: magmhcr@gmail.com

ORCID: 0000-0001-7038-8301 